\title{
A Calibrated Imputation Method for Secondary Data Analysis of
}

\section{Survey Data.}

\author{
Damião Nóbrega Da Silva*1 | Li-Chun Zhang ${ }^{2,3,4}$
}

\author{
${ }^{1}$ Departamento de Estatística, Universidade \\ Federal do Rio Grande do Norte, Natal, \\ RN, Brazil \\ ${ }^{2}$ Southampton Statistical Sciences Research \\ Institute, University of Southampton, \\ Southampton, Hampshire, UK \\ ${ }^{3}$ Statistisk sentralbyrå, Oslo, Norway \\ ${ }^{4}$ University of Oslo, Oslo, Norway

\section{Correspondence} \\ *Damião Nóbrega Da Silva, Departamento \\ de Estatística, Centro de Ciências Exatas e \\ da Terra, Universidade Federal do Rio \\ Grande do Norte, Natal, RN, 59078-970 \\ Brazil.Email: damiao@ccet.ufrn.br
}

Funding Information

This research was supported by the Brazilian Research Council (CNPq), Grant Number: $211518 / 2013-1$

\begin{abstract}
Summary
In practical survey sampling, missing data are unavoidable due to nonresponse, rejected observations by editing, disclosure control or outlier suppression. We propose a calibrated imputation approach so that valid point and variance estimates of the population (or domain) totals can be computed by the secondary users using simple complete-sample formulae. This is especially helpful for variance estimation, which generally require additional information and tools that are unavailable to the secondary users. Our approach is natural for continuous variables, where the estimation may be either based on reweighting or imputation, including possibly their outlier-robust extensions. We also propose a multivariate procedure to accommodate the estimation of the covariance matrix between estimated population totals, which facilitates variance estimation of the ratios or differences among the estimated totals. We illustrate the proposed approach using simulation data in supplementary materials that are available online.
\end{abstract}

\section{KEYWORDS:}

analysis of incomplete data, item nonresponse, missing data, variance estimation

\section{1 | INTRODUCTION}

In the preparation of survey data for use by secondary analysts, some or all of the sample units are usually assigned estimation weights that can be applied to all the survey variables. In addition to these weights, imputed values may be needed for the units that are subjected to item missingness. It is often possible to choose the imputed values for each survey variable so that, together with the observed and retained values of this variable, the corresponding population total can be estimated by weighting as if

\footnotetext{
${ }^{0}$ Abbreviations: ANA, anti-nuclear antibodies; APC, antigen-presenting cells; IRF, interferon regulatory factor
} 
the sample were completely observed. However, applying standard complete-sample variance estimator formulae to the same imputed sample would generally cause bias (see, e.g., Wolter 2007, pp. 419, 421).

Variance estimation in the presence of imputed data needs to appropriately account for the underlying data generation mechanism. Some common techniques are Fay's reverse framework (e.g. Fay, 1991, 1992, Shao \& Steel, 1999, Kim \& Rao, 2009), two-phase sampling (Särndal, 1992, Deville \& Särndal, 1994b) and replication methods (Rao \& Shao, 1992, Rao, 1996, Shao \& Sitter, 1996, Chen, Rao, \& Sitter 2000). To choose and apply any of these methods may be difficult for secondary users who are non-specialists, even impossible if the relevant information about the sampling design and data processing is lacking.

The needs for easier secondary analyses ensuring that different users of the imputed data could obtain the same results by simple estimation methods have long been recognized (Kalton \& Kasprzyk 1982). Early work under this estimation perspective for the imputed data was addressed by Lanke (1983), Sedransk (1985) and Kim (2001a). Some later works considered the use of constrained or calibrated imputation for point estimation in different situations (Chambers \& Ren, 2004, Beaumont 2005: Chauvet, Deville, \& Haziza, 2011, Gelein, Haziza, \& Causeur, 2014). Multiple imputation (Rubin, 1978a, 1987b, 1996c, Rubin \& Schenker, 1986) and fractional imputation (Kim \& Fuller, 2004, Fuller \& Kim, 2005) are two methods based on multiply imputed values. For instance, provided the multiple imputation procedure is proper or the congeniality condition (Meng 1994) holds, one can compute valid point and variance estimates by combining the results obtained from applying standard complete-sample formulae to each imputed sample.

In this paper, we propose a calibrated imputation approach that allows for valid point and variance estimation of the population and domain totals (or means), by applying simple complete-sample formulae to the imputed sample. Although this accommodates a more limited scope than multiple or fractional imputation, secondary users can achieve the intended analyses based on a single imputed dataset using standard software. Moreover, we provide a multivariate procedure for the estimation of a vector of totals (or means) and the associated covariance matrix, using simple complete-sample formulae. This allows one to estimate the variance of ratios or differences of the estimated totals. Finally, the proposed approach has also benefits to the data producer, such as avoiding the dissemination of multiply imputed datasets, the freedom to choose a suitable inference outlook and apply different missing data treatments from one variable to another.

The rest of the paper is organized as follows. The proposed approach is outlined in Section 2. We explain in Section 3 how it can be applied in some common situations of reweighting and imputation-based estimation, as well as domain estimation 
and estimation under stratified multistage sampling. The multivariate calibrated imputation procedure is described in Section 4.

Some concluding remarks and future research topics are given in Section 5.

\section{2 | CALIBRATION OF A SINGLE VARIABLE}

\section{1 | Estimation Setup}

Consider a finite population of $N$ units denoted by $U=\{1,2, \ldots, N\}$ and let $Y_{N}=\left(y_{1}, \ldots, y_{N}\right)$, where $y_{k}$ is the value of a survey variable $y$ for the $k$ th unit, $k \in U$. Let $A$ be a sample from $U$ selected by a probability sampling design $p\left(I_{N}\right)$, where $I_{N}=\left(i_{1}, \ldots, i_{N}\right)$ and $i_{k}=1$ if the $k$ th unit is selected to the sample $A$ and $i_{k}=0$ otherwise, and let $R_{N}=\left(r_{1}, \ldots, r_{N}\right)$, where $r_{k}=1$ if $y_{k}$ is observed and $r_{k}=0$ if the $y_{k}$ is unobserved or rejected during data processing $(k \in U), A_{r}=\left\{k: k \in A, r_{k}=1\right\}$ be the set of units for which the observations are to be preserved and $A_{m}=\left\{k: k \in A, r_{k}=0\right\}$ be the set for which imputation is needed. We assume the missing information of the variable $y$ for the units in $A_{m}$ is filled in by imputation and denote the corresponding imputed values by $\left\{y_{k}^{*}: k \in A_{m}\right\}$. We assume, in addition, that the imputed dataset will be accompanied by a set of survey weights $\left\{w_{k}: k \in A\right\}$, as for instance, the inverse of the inclusion probabilities (Horvitz \& Thompson, 1952), or weights suitably calibrated for auxiliary population totals (Deville \& Särndal, 1992a).

Suppose it is of interest to estimate the population total of the variable $y$, that is $t_{y}=\sum_{k \in U} y_{k}$. When it comes to completesample estimation of $t_{y}$ using the imputed data $\left\{\left(w_{k}, y_{k}^{*}\right): k \in A\right\}$, where $y_{k}^{*}$ is the value for unit $k$ in the imputed full sample $A$ with $y_{k}^{*}=y_{k}$ for $k \in A_{r}$, a natural and simple choice for the imputed estimator is

$$
\hat{t}_{y I}=\sum_{k \in A} w_{k} y_{k}^{*}
$$

Statistical properties of $\hat{t}_{y I}$ are studied by adopting an inference approach for the imputed data, which is usually specified by postulating explicitly a model for the distribution of the response indicators or a superpopulation model for the values of the variables of interest in the population (Haziza 2009. pp. 222-223). The properties of $\hat{t}_{y I}$ are then evaluated with respect to the joint distribution of the sampling design and the assumed model, allowing the unconditional variance of the imputed estimator to be decomposed into variance components which, when estimated, lead to the estimated variance of $\hat{t}_{y I}$. 
Here we consider instead the estimation of the variance of the imputed estimator $\hat{t}_{y I}$ by means of the complete-sample estimator

$$
\hat{v}_{F}\left(\hat{t}_{y I}\right) \equiv \frac{n}{n-1} \sum_{k \in A}\left(u_{k}^{*}-\bar{u}^{*}\right)^{2}
$$

where $u_{k}^{*}=w_{k} y_{k}^{*}(k \in A)$ and $\bar{u}^{*}=\sum_{k \in A} u_{k}^{*} / n=\hat{t}_{y I} / n$, which amounts to the with-replacement pps sampling variance formula and, hence, may be computed more easily by secondary users using standard software. For example, when $w_{k}=N / n$ then $\hat{t}_{y I}=N \bar{y}_{I}$ and $\operatorname{Var}\left(\hat{t}_{y I}\right)=N^{2} s_{y I}^{2} / n$, where $\bar{y}_{I}$ and $s_{y I}^{2}$ are the sample mean and variance of the imputed variable.

Clearly, naive application of estimators (1) and (2) would lead to incorrect inference generally. In order for these estimators to yield valid estimates, the imputed values need to be created in a controlled manner, as it will be discussed in the next section.

\section{2 | The calibrated imputation approach}

The main goal of the following calibration method for the imputed data is to provide imputed values $y_{k}^{*}$ so that the completesample estimators (1) and (2) satisfy

$$
\hat{t}_{y I} \equiv \sum_{k \in A} w_{k} y_{k}^{*}=\hat{t}_{y 0}, \quad \hat{v}_{F}\left(\hat{t}_{y I}\right) \equiv \frac{n}{n-1} \sum_{k \in A}\left(w_{k} y_{k}^{*}-\hat{t}_{y I} / n\right)^{2}=\hat{v}_{y 0},
$$

where $\hat{t}_{y 0}$ and $\hat{v}_{y 0}$ are valid target estimates for the population total and its corresponding variance estimate. The method requires the data producer to choose and calculate such targets for the variable specified, as well as to calibrate the imputed values to attain the conditions in (3). These targets should incorporate all the aspects of the sampling design, response mechanism and inference approach for the imputed estimator. However, as a benefit of the calibration method, the suitability of these target estimates is a matter of concern only for the data producer and not for the secondary users, who are no longer exposed to the theoretical and computational complications involved.

The calibration method can be described by the following two-step algorithm.

\section{Calibration algorithm:}

Step 1 (Imputation): Using a standard imputation procedure, obtain a set of initial imputed values $\left\{\tilde{y}_{k}: k \in A_{m}\right\}$. For each $\tilde{y}_{k}$, obtain a corresponding adjusted imputed value $\hat{y}_{k}$ so that

$$
\sum_{k \in A_{m}} w_{k} \hat{y}_{k}=\hat{t}_{y 0}-\sum_{k \in A_{r}} w_{k} y_{k}
$$


Step 2 (Calibration): For each $k \in A_{r}$, set $u_{k}^{*}=w_{k} y_{k}$. For $k \in A_{m}$, obtain an imputed value $u_{k}^{*}$ by a minimal adjustment to $\hat{u}_{k}=w_{k} \hat{y}_{k}$, where $\hat{y}_{k}$ is computed in Step 1, so that

$$
\sum_{k \in A_{m}} u_{k}^{*}=\hat{t}_{y 0}-\sum_{k \in A_{r}} w_{k} y_{k}
$$

and

$$
\sum_{k \in A_{m}} u_{k}^{* 2}=\frac{n-1}{n} \hat{v}_{y 0}+\frac{1}{n} \hat{t}_{y 0}^{2}-\sum_{k \in A_{r}} w_{k}^{2} y_{k}^{2}
$$

where $\hat{t}_{y 0}$ and $\hat{v}_{y 0}$ are the targets in (3). Take $y_{k}^{*}=u_{k}^{*} / w_{k}$ for $k \in A$.

The algorithm initiates in Step 1 by choosing an imputation scheme to provide preliminary imputed values $\hat{y}_{k}$, for $k \in A_{m}$, such that applying (1) with these values yields $\hat{t}_{y 0}$. Provided the initial imputed values $\tilde{y}_{k}\left(k \in A_{m}\right)$ already yields $\hat{t}_{y 0}$ by (1), one can simply take $\hat{y}_{k}=\tilde{y}_{k}$, for $k \in A_{m}$. An example is given in Section 3.1. Otherwise, the $\tilde{y}_{k}$ values need to be adjusted. One simple ratio adjustment of the initial imputed values is

$$
\hat{y}_{k}=\frac{\left(\hat{t}_{y 0}-\sum_{\ell \in A_{r}} w_{\ell} y_{\ell}\right)}{\sum_{\ell \in A_{m}} w_{\ell} \tilde{y}_{\ell}} \tilde{y}_{k} \quad\left(k \in A_{m}\right),
$$

which is a special case of the reverse calibration approach of Chambers \& Ren (2004), originally proposed for the estimation of $t_{y}$ in the presence of survey outliers. Then, in Step 2, the calibration of the imputed values is made. Optimal imputed values that are calibrated to (5) and (6) could be computed in closed-form by applying Theorem 1 below. The proof of this theorem is shown in the Appendix.

Theorem 1. Consider initial values $\hat{a}_{k}$ and $d_{k}>0$ for all $k$ in a non-null set $D \subset A$. Suppose $\sum_{k \in D} d_{k} \hat{a}_{k}=t_{1}$ for some fixed constant $t_{1}$ and $\sum_{k \in D} d_{k}\left(\hat{a}_{k}-t_{1} / t_{0}\right)^{2}>0$, where $t_{0}=\sum_{k \in D} d_{k}>0$. Let $t_{2}>t_{1}^{2} / t_{0}$ be a fixed constant . Then, the adjusted $a_{k}$ values that minimize $\Delta=\sum_{k \in D} d_{k}\left(a_{k}-\hat{a}_{k}\right)^{2}$ subjected to the constraints

$$
\sum_{k \in D} d_{k} a_{k}=t_{1}, \quad \sum_{k \in D} d_{k} a_{k}^{2}=t_{2},
$$

are given by

$$
a_{k}=t_{1} / t_{0}+\beta\left(\hat{a}_{k}-t_{1} / t_{0}\right),
$$

where

$$
\beta=\left(\frac{t_{2}-t_{1}^{2} / t_{0}}{\hat{t}_{2}-t_{1}^{2} / t_{0}}\right)^{1 / 2}
$$

and $\hat{t}_{2}=\sum_{k \in D} d_{k} \hat{a}_{k}^{2}$. 
The optimal calibrated imputed values $y_{k}^{*}$ of Step 2 are obtained as follows. First, we take the values of the calibration conditions $t_{1}$ and $t_{2}$ of (8) as the right-hand sides of (5) and (6), namely

$$
t_{1}=\hat{t}_{y 0}-\sum_{k \in A_{r}} w_{k} y_{k}
$$

and

$$
t_{2}=(n-1) \hat{v}_{y 0} / n+\hat{t}_{y 0}^{2} / n-\sum_{k \in A_{r}} w_{k}^{2} y_{k}^{2} .
$$

Then, we set $D=A_{m}, d_{k}=1$ and $\hat{a}_{k}=\hat{u}_{k}=w_{k} \hat{y}_{k}$ for all $k \in A_{m}$, where the $\hat{y}_{k}\left(k \in A_{m}\right)$ are obtained in Step 1 . Thus, it follows from (9] that the $u_{k}^{*}$ values of Step 2 are

$$
u_{k}^{*} \equiv a_{k}=t_{1} / m+\hat{\beta}\left(\hat{u}_{k}-t_{1} / m\right)\left(k \in A_{m}\right)
$$

where $t_{1}$ is defined in $[10$ and

$$
\hat{\beta}=\left\{\frac{(n-1) \hat{v}_{y 0} / n-\sum_{k \in A_{r}}\left(u_{k}-\hat{t}_{y 0} / n\right)^{2}-m\left(\hat{t}_{y 0} / n-t_{1} / m\right)^{2}}{\sum_{k \in A_{m}}\left(\hat{u}_{k}-t_{1} / m\right)^{2}}\right\}^{1 / 2} .
$$

The resulting calibrated imputed variable is

$$
y_{k}^{*}=\left\{\begin{array}{l}
y_{k}, \quad k \in A_{r}, \\
u_{k}^{*} / w_{k}, k \in A_{m} .
\end{array}\right.
$$

Remark 1. The calibrated imputation method in (12) does not modify the observed values for units in the respondent set $\left(A_{r}\right)$. The values that are actually modified are the calibrated $y_{k}^{*}=u_{k}^{*} / w_{k}$ values $\left(k \in A_{m}\right)$, where the $u_{k}^{*}$ values minimize the squared distance to the imputed values $\hat{u}_{k}=w_{k} \hat{y}_{k}\left(k \in A_{m}\right)$ obtained in Step 1 , that is, $\Delta=\sum_{k \in A_{m}}\left(u_{k}^{*}-\hat{u}_{k}\right)^{2}$. The resulting $u_{k}^{*}$ values are obtained analytically as the "best" linear predictor of $u_{k}$ based on the $\hat{u}_{k}\left(k \in A_{m}\right)$, where the slope $\hat{\beta}$ of the regression line, given in (11), dictates how the empirical variance of the $u_{k}^{*}$ relates to that of the $\hat{u}_{k}\left(k \in A_{m}\right)$. In practice, unless the $\hat{y}_{k}$ values are created to have greater empirical variance over $A_{m}$ than $A_{r}$, one may expect $\hat{\beta}>1$. This is because the formula (2) is ostensibly aimed at a variance of the order $n^{-1}$, whereas the target $\hat{v}_{y 0}$ is generally aimed at a variance of the order $r^{-1}$, where $r$ is the size of $A_{r}$. Thus, in order for the two to be equal to each other, the imputed $y_{k}^{*}$ values will need to have greater variation over $A_{m}$ than the observed $y_{k}$ over $A_{r}$.

Remark 2. Given the set of missing units $A_{m}$, the application of Theorem 1 to obtain the optimal solution 11 requires that

$$
\hat{v}_{y 0}>\frac{n}{n-1}\left\{\sum_{k \in A_{r}}\left(u_{k}-\hat{t}_{y 0} / n\right)^{2}+m\left(t_{1} / m-\hat{t}_{y 0} / n\right)^{2}\right\}
$$


and

$$
\sum_{k \in A_{m}}\left(\hat{u}_{k}-t_{1} / m\right)^{2}>0
$$

Comparing (13) to (2), it is readily seen that, for the solution to the optimization problem in Step 2 to exist, the target estimate $\hat{v}_{y 0}$ needs to be larger than the full-sample variance estimate (2) that would have been obtained had the missing values been imputed by the common value $t_{1} / m$. The second condition (14) demands that the sampling weights and the imputation scheme are such that the $\hat{u}_{k}=w_{k} \hat{y}_{k}$ values are different from $t_{1} / m$ for at least one $k \in A_{m}$. This is not the case when mean imputation is used at Step 1 to fill in the missing values of an equal probability sample. In such a situation, the proposed approach could still be applied by adding some initial zero-mean noise to each mean imputed value. The calibration constraints ensure that this added variability will not affect the variance of the imputed estimator.

\section{3 | SOME APPLICATIONS}

We explain below how the two-step approach and Theorem 1 proposed in Section 2 can be applied in some general situations, which comprise reweighting and imputation-based estimation, as well as domain estimation and estimation under stratified multistage sampling.

\section{1 | Ratio imputation}

Suppose that, in addition to the survey variable $y$, there is an auxiliary variable $x$ which is not affected by nonresponse. Assume a population ratio model $\xi$ of the pairs $\left\{\left(x_{k}, y_{k}\right): k \in U\right\}$, under which

$$
E_{\xi}\left(y_{k} \mid x_{k}\right)=\beta_{0} x_{k}, \quad \operatorname{Var}_{\xi}\left(y_{k} \mid x_{k}\right)=\sigma^{2} x_{k},
$$

for some unknown parameters $\beta_{0}$ and $\sigma^{2}$. By ratio imputation under the model $\xi$, the missing $y_{k}$ values are imputed as

$$
\tilde{y}_{k}=\hat{\beta}_{0 r} x_{k} \quad\left(k \in A_{m}\right),
$$


where $\hat{\beta}_{0 r}=\sum_{k \in A_{r}} w_{k} y_{k} / \sum_{k \in A_{r}} w_{k} x_{k}$, and $w_{k}=1 / \pi_{k}$, and $\pi_{k}$ is the sample inclusion probability, for $k \in A$. The resulting imputed estimator of the population total $t_{y}$ is

$$
\hat{t}_{y 0}=\sum_{k \in A_{r}} w_{k} y_{k}+\sum_{k \in A_{m}} w_{k} \tilde{y}_{k}=\hat{\beta}_{0 r} \hat{t}_{x}
$$

where $\hat{t}_{x}=\sum_{k \in A} w_{k} x_{k}$ is the Horvitz-Thompson estimator (Horvitz \& Thompson. 1952) of the population total $t_{x}=\sum_{k \in U} x_{k}$. Mean imputation is a special case of ratio imputation with $x_{k}=1$ for all $k \in A$, by which the imputed estimator $\hat{t}_{y 0}$ reduces to $\hat{t}_{y 0}=N \bar{y}_{r}$. Under the conditions of Theorem 1 of $\operatorname{Kim} \& \operatorname{Rao}(2009)$, a design and model consistent estimator of the variance of $\hat{t}_{y 0}$ can be expressed as

$$
\hat{v}_{y 0}=\hat{v}_{1}+\hat{v}_{2}
$$

where

$$
\begin{gathered}
\hat{v}_{1}=\sum_{k \in A} \sum_{\ell \in A} \frac{\left(\pi_{k \ell}-\pi_{k} \pi_{\ell}\right)}{\pi_{k \ell}} w_{k} \hat{\eta}_{k} w_{\ell} \hat{\eta}_{\ell}, \quad \hat{v}_{2}=\left(\frac{\hat{t}_{x}}{\hat{t}_{x r}}\right)^{2} \sum_{k \in A_{r}} w_{k} \hat{e}_{k}^{2}, \\
\hat{\eta}_{k}=\hat{\beta}_{0 r} x_{k}+\frac{\hat{t}_{x}}{\hat{t}_{x r}} r_{k} \hat{e}_{k} \quad(k \in A)
\end{gathered}
$$

$\hat{t}_{x r}=\sum_{k \in A_{r}} w_{k} x_{k}$ and $\hat{e}_{k}=y_{k}-\hat{\beta}_{0 r} x_{k}$

However, to compute $\hat{v}_{1}$, the secondary user needs to have access to the matrix of the second-order inclusion probabilities $\left\{\pi_{k \ell}: k \ell \in A\right\}$, which are almost never disseminated together with the imputed sample. The proposed approach avoids this complication. To calibrate the ratio imputed values $\tilde{y}_{k}=\hat{\beta}_{0 r} x_{k}\left(k \in A_{m}\right)$, we notice that $\hat{y}_{k}=\tilde{y}_{k}$ already satisfies Step 1 , since $t_{1}=\hat{\beta}_{0 r} \hat{t}_{x m}$ in Theorem 1 For Step 2, by $(12)$ and $(11)$, the calibrated imputed values are

$$
y_{k}^{*}=u_{k}^{*} / w_{k},
$$

where $u_{k}^{*}=w_{k} y_{k}\left(k \in A_{r}\right)$, and for $k \in A_{m}$,

$$
u_{k}^{*}=\frac{\hat{\beta}_{0 r} \hat{t}_{x m}}{m}+\hat{\beta} \hat{\beta}_{0 r}\left(w_{k} x_{k}-\frac{\hat{t}_{x m}}{m}\right) \quad\left(k \in A_{m}\right)
$$

and

$$
\hat{\beta}^{2}=\frac{\frac{(n-1)}{n}\left(\hat{v}_{1}+\hat{v}_{2}\right)-\sum_{k \in A_{r}}\left(w_{k} y_{k}-\frac{\hat{\beta}_{0 r} \hat{t}_{x}}{n}\right)^{2}-m \hat{\beta}_{0 r}^{2}\left(\frac{\hat{t}_{x}}{n}-\frac{\hat{t}_{x m}}{m}\right)^{2}}{\hat{\beta}_{0 r}^{2} \sum_{k \in A_{m}}\left(w_{k} x_{k}-\frac{\hat{t}_{x m}}{m}\right)^{2}} .
$$

In the case of mean imputation and simple random sampling without replacement, (15) reduces to

$$
\hat{v}_{y 0}=N^{2}\left(\frac{1}{r}-\frac{1}{N}\right) s_{y r}^{2}
$$


where $s_{y r}^{2}=\sum_{k \in A_{r}}\left(y_{k}-\bar{y}_{r}\right)^{2} /(r-1)$ and $\bar{y}_{r}$ is the observed respondent mean.

\section{2 | Domain estimation}

As a realistic setting for domain total estimation, in addition to the population total, consider a domain population partition $U=U_{1} \cup \cdots \cup U_{D}$. Let the population total of domain $U_{d}$ be

$$
t_{d y}=\sum_{k \in U_{d}} y_{k}=\sum_{k \in U} \delta_{k d} y_{k},
$$

where the domain indicator $\delta_{k d}, \delta_{k d}=1$ if $k \in U_{d}$ and $\delta_{k d}=0$ otherwise, is observed for all units in the sample $A(d=$ $1, \ldots, D)$. Let $\hat{t}_{d y}$ be the target domain total estimator and $\hat{v}_{d y}$ its variance estimate. Domain estimation can be handled by separate calibration for each domain by the producer and application of the domain complete-data formulae by the secondary users, yielding $\hat{t}_{d y I}=\hat{t}_{d y}$ and $\hat{v}_{F}\left(\hat{t}_{d y I}\right)=\hat{v}_{d y}$, as explained in Section 2 .

However, one is still interested in estimating the population total, in addition to the domain totals. Directly applying the complete-sample formula (1) to the domain-calibrated imputed sample would correctly estimate the population total. One can combine the domain variance estimates, as if the sampling were stratified by the domains. However, the resulting variance estimate is incorrect even when the domain total estimators are independent of each other, due to the additional term

$$
v_{b}=\frac{n}{n-1} \sum_{d=1}^{D} n_{d}\left(\hat{t}_{d y I} / n_{d}-\hat{t}_{y 0} / n\right)^{2}=\frac{n^{2}}{n-1} V_{n}\left(\hat{t}_{d y I} / n_{d}\right)
$$

where $V_{n}\left(\hat{t}_{d y I} / n_{d}\right)$ is the variance of $\hat{t}_{d y I} / n_{d}$ with respect to the empirical sample domain distribution function $\left(n_{1} / n, \ldots, n_{D} / n\right)$, since

$$
V_{n}\left(\hat{t}_{d y I} / n_{d}\right)=\sum_{d=1}^{D} \frac{n_{d}}{n}\left(\hat{t}_{d y I} / n_{d}-\hat{t}_{y 0} / n\right)^{2} \quad \text { and } \quad \hat{t}_{y 0} / n=E_{n}\left(\hat{t}_{d y I} / n_{d}\right)=\sum_{d=1}^{D} \frac{n_{d}}{n}\left(\hat{t}_{d y I} / n_{d}\right) .
$$

We propose to introduce a domain estimation effect factor, denoted by $\gamma$, and use

$$
\hat{v}_{F}\left(\hat{t}_{y I}\right)=\gamma^{2} \frac{n}{n-1} \sum_{k \in A}\left(w_{k} y_{k}^{*}-\hat{t}_{y 0} / n\right)^{2}=\hat{v}_{y 0}
$$

The factor $\gamma$ can be calculated after domain-calibrated imputation, and disseminated together with imputed sample.

In the separate domain calibration above, $\hat{v}_{F}\left(\hat{t}_{d y I}\right)$ is built on the squared errors around $\hat{t}_{d y I} / n_{d}$. Consider using another complete-sample formula $\hat{v}_{F}\left(\hat{t}_{d y I}\right)$, built around $\hat{t}_{y I} / n$ instead, where

$$
\hat{v}_{F}\left(\hat{t}_{d y I}\right)=\frac{n_{d}}{n_{d}-1} \sum_{k \in A} \delta_{k d}\left(w_{k} y_{k d}^{*}-\hat{t}_{y 0} / n\right)^{2} .
$$


We need to extend the calibration constraints as follows:

$$
\begin{cases}\hat{t}_{d y I}=\sum_{k \in A} \delta_{k d} w_{k} y_{k}^{*}=\hat{t}_{d y} & \text { for } d=1, \ldots, D \\ \hat{v}_{F}\left(\hat{t}_{d y I}\right)=\frac{n_{d}}{n_{d}-1} \sum_{k \in A} \delta_{k d}\left(w_{k} y_{k d}^{*}-\hat{t}_{y 0} / n\right)^{2}=\hat{v}_{d y} & \text { for } d=1, \ldots, D \\ \hat{v}_{F}\left(\hat{t}_{y I}\right)=\gamma^{2} \frac{n}{n-1} \sum_{k \in A}\left(w_{k} y_{k}^{*}-\hat{t}_{y 0} / n\right)^{2}=\hat{v}_{y 0} . & \end{cases}
$$

In other words, we use $\delta_{k d}$ to identify the relevant observations for domain estimation, including the special case of $U_{d}=U$ and $\delta_{k d} \equiv 1$, and use $\hat{t}_{y 0} / n$ in all the ultimate variance estimators, including domain variance estimation. We refer to (19) as the centred domain calibration approach.

Minimum adjustments of $\left\{\hat{y}_{k} ; k \in A_{m}\right\}$ from Step 2 of the proposed approach can be achieved by Theorem 1 as well. To focus the idea, suppose negligible $1 / n$ and $1 / n_{d}$. Let $\left\{u_{k}^{*} ; k \in A_{m d}\right\}$ be the calibrated imputations in domain $d$, given by

$$
u_{k}^{*}=t_{1 d} / m+\beta_{d}\left(\hat{u}_{k}-t_{1 d} / m\right)
$$

where $t_{1 d}=\hat{t}_{d y}-\sum_{k \in A_{r d}} w_{k} y_{k}$ is the constrained total of $u_{k}^{*}=w_{k} y_{k}^{*}$ in $A_{m d}$. However, instead of choosing $\beta_{d}$ such that

$$
\beta_{d}^{2} \sum_{k \in A_{m d}}\left(\hat{u}_{k}-\frac{t_{1 d}}{m}\right)^{2}=\hat{v}_{d y}-\sum_{k \in A_{r d}}\left(u_{k}-\frac{\hat{t}_{d y}}{n_{d}}\right)^{2}-m_{d}\left(\frac{\hat{t}_{d y}}{n_{d}}-\frac{t_{1 d}}{m}\right)^{2},
$$

as under separate domain calibration, we should now choose $\beta_{d}$ such that

$$
\beta_{d}^{2} \sum_{k \in A_{m d}}\left(\hat{u}_{k}-\frac{t_{1 d}}{m}\right)^{2}=\hat{v}_{d y}-\sum_{k \in A_{r d}}\left(u_{k}-\frac{\hat{t}_{y 0}}{n}\right)^{2}-m_{d}\left(\frac{\hat{t}_{y 0}}{n}-\frac{t_{1 d}}{m}\right)^{2} .
$$

This allows us to estimate the domain variance $\hat{v}_{d y}$ as in 19 . The domain estimation effect factor $\gamma$ can be calculated afterwards to satisfy (19). The conditions for the existence of solution are formally the same as discussed in Section 2.2. Provided domainspecific calibration, it is feasible as long as $\hat{t}_{y 0} / n$ does not differ too much from $\hat{t}_{d y} / n_{d}$ in the different domains.

In practice one may be interested in multiple sets of (overlapping) domains. For example, a user may want to have estimates by region as well as estimates by industry. Insofar as the need is known in advance, the producer can apply the approach above to the 'atomic domains', which arise from crossing region and industry. In addition to the separate atomic-domain calibrated sample, one can supply a domain estimation factor for the population total, a set of domain estimation factors for each of the regions, and another set of factors for each industry. 


\subsection{Stratified Multistage Sampling}

Let the population $U$ be partitioned into $H$ strata of $n$ primary sampling units (PSUs), where a sample $A_{h}$ of $n_{h}$ PSUs is selected separately within the $h$ th stratum $\left(h=1, \ldots, H ; n_{1}+\cdots+n_{H}=n\right)$. From each PSU in $A_{h}$, additional stages of sampling are undertaken until the selection of the ultimate sampling units (USUs). Let $w_{i}, y_{i}$ and $r_{i}$ be, respectively, the weight, the $y$ value and the response indicator for the $i$ th USU. Let $A_{h k}$ be the set of USUs in the $k$ th selected PSU of the $h$ th stratum, where $A_{r h k}=\left\{i: i \in A_{h k}, r_{i}=1\right\}$ and $A_{m h k}=\left\{i: i \in A_{h k}, r_{i}=0\right\}$.

By setting $y_{i}^{*}=y_{i}$ if $r_{i}=1$ and letting $y_{i}^{*}$ be the calibrated imputation value if $r_{i}=0$, the imputed estimate of the population total $t_{y}$ can be written as $\hat{t}_{y I}=\sum_{h=1}^{H} \hat{t}_{y I h}$, where $\hat{t}_{y I h}=\sum_{k \in A_{h}} u_{h k}^{*}$ and $u_{h k}^{*}=\sum_{i \in A_{h k}} w_{i} y_{i}^{*}=\sum_{i \in A_{r h k}} w_{i} y_{i}+\sum_{i \in A_{m h k}} w_{i} y_{i}^{*}$. For calibrated imputation that enables (3), we can apply Theorem 1 and the 2-step approach directly at the level of USUs, ignoring the clustering structure of the multistage sampling.

Survey data analysis softwares (such as STATA, R, SAS) commonly use the stratified ultimate variance formula for variance estimation. It is therefore convenient if the secondary user can simply input the imputed sample, and let the software carry on as usual. Thus, as another possibility of full-sample variance estimator, we consider

$$
\hat{v}_{F}\left(\hat{t}_{y I}\right)=\sum_{h=1}^{H} \frac{n_{h}}{n_{h}-1} \sum_{k \in A_{h}}\left(u_{h k}^{*}-\bar{u}_{h}^{*}\right)^{2},
$$

where $\bar{u}_{h}^{*}=\sum_{k \in A_{h}} u_{h k}^{*} / n_{h}=\hat{t}_{y I h} / n_{h}$. This choice fits naturally with the standard approach of ultimate-cluster variance estimation under stratified multistage sampling (e.g. Skinner. 1989, Section 2.13).

Given $\hat{t}_{y 0 h}$ for the population total in the $h$ th stratum and its associated variances $\hat{v}_{y 0 h}=\hat{v}\left(\hat{t}_{y 0 h}\right)(h=1, \ldots, H)$, consider the problem of finding the values $y_{j}^{*}$ starting with $\tilde{y}_{j}, j \in \cup_{k \in A_{h}} A_{m h k}$, so that

$$
\begin{aligned}
& \hat{t}_{y I h}=\sum_{k \in A_{h}} u_{h k}^{*}=\hat{t}_{y 0 h}, \\
& \sum_{k \in A_{h}}\left(u_{h k}^{*}-\bar{u}_{h}^{*}\right)^{2}=\sum_{k \in A_{h}} u_{h k}^{* 2}-\hat{t}_{y 0 h}^{2} / n_{h}=\left(n_{h}-1\right) \hat{v}_{y 0 h} / n_{h} .
\end{aligned}
$$

We propose to obtain a solution of this problem in two stages. First, the initial imputed PSU totals are adjusted minimally subject to the two constraints above, yielding the adjusted PSU total $u_{h k}^{*}$. Second, the initial imputed values $\tilde{y}_{j}$ are adjusted, separately within each PSU, to agree with the corresponding calibrated PSU total from the first step.

For the first stage, we can apply Theorem 1 within the $h$ th stratum similarly as in Section 2 . Let $A_{h 0}=\left\{k \in A_{h}: \#\left(A_{m h k}\right)=\right.$ $0\}, \hat{u}_{h k}=u_{h k}$ for $k \in A_{h 0}$ and $\hat{u}_{h k}=\tilde{u}_{h k}\left(\hat{t}_{y 0 h}-\sum_{k \in A_{h 0}} u_{h k}\right) /\left(\sum_{\ell \in A_{h} \backslash A_{h 0}} \tilde{u}_{h \ell}\right)$ for $k \in A_{h} \backslash A_{h 0}$. Then, take $D=D_{h}=A_{h} \backslash A_{h 0}$, 
$d_{k}=d_{h k}=1, \hat{a}_{k}=\hat{a}_{h k}=\hat{u}_{h k}, t_{0}=\sum_{k \in A_{h} \backslash A_{h 0}} d_{k} \equiv m_{h}, t_{1}=t_{1 h}=\hat{t}_{y 0 h}-\sum_{k \in A_{h 0}} u_{h k}$ and $t_{2}=t_{2 h}=\left(n_{h}-1\right) \hat{t}_{y 0 h} / n_{h}+$ $\hat{t}_{y 0 h}^{2} / n_{h}-\sum_{k \in A_{h 0}} u_{h k}^{2}$. For each $h=1, \ldots, H$, the optimal solution that minimizes the squared distance $\Delta_{h}=\sum_{A_{h}}\left(u_{h k}^{*}-\hat{u}_{h k}\right)^{2}=$ $\sum_{A_{h} \backslash A_{h 0}}\left(u_{h k}^{*}-\hat{u}_{h k}\right)^{2}$ subject to (20) are given by $u_{h k}^{*}=u_{h k}$ for $k \in A_{h 0}$, and

$$
u_{h k}^{*}=\frac{\hat{t}_{1 h}}{m_{h}}+\hat{\beta}_{h}\left(\hat{u}_{h k}-\frac{\hat{t}_{1 h}}{m_{h}}\right)
$$

for $k \in A_{h} \backslash A_{h 0}$, where

$$
\hat{\beta}_{h}=\left\{\frac{\left(n_{h}-1\right) \hat{v}_{y 0 h} / n_{h}-\sum_{A_{h 0}}\left(u_{h k}-\hat{t}_{y 0 h} / n_{h}\right)^{2}-m_{h}\left(\hat{t}_{y 0 h} / n_{h}-t_{1 h} / m_{h}\right)^{2}}{\sum_{k \in A_{h} \backslash A_{h 0}}\left(\hat{u}_{h k}-t_{1 h} / m_{h}\right)^{2}}\right\}^{\frac{1}{2}} .
$$

Having thus obtained $u_{h k}^{*}$, we adjust the $\tilde{y}_{i}$ 's separately within each PSU so that $u_{h k}^{*}=\sum_{i \in A_{r h k}} w_{i} y_{i}+\sum_{i \in A_{m h k}} w_{i} y_{i}^{*}$, which is a single constraint. For given $h$ and $k \in A_{h} \backslash A_{h 0}$, the values $y_{i}^{*}$ that minimize the distance $\sum_{i \in A_{m h k}}\left(y_{i}^{*}-\tilde{y}_{i}\right)^{2} / 2$ subject to $\sum_{j \in A_{\text {mhk }}} w_{i} y_{i}^{*}=u_{h k}^{*}-\sum_{i \in A_{r h k}} w_{i} y_{i} \equiv u_{h k 0}$ are

$$
y_{i}^{*}=\tilde{y}_{i}\left\{1+\left(\frac{w_{i}}{\tilde{y}_{i}}\right) \frac{\left(u_{h k 0}-\sum_{i \in A_{m h k}} w_{i} \tilde{y}_{i}\right)}{\sum_{i \in A_{m h k}} w_{i}^{2}}\right\} \quad\left(i \in A_{m h k}\right) .
$$

\section{4 | CALIBRATION OF MULTIPLE VARIABLES}

Let $\boldsymbol{y}_{k}=\left(y_{k 1}, \ldots, y_{k p}\right)^{\top}$ denote a $p$-dimensional vector of values for the $k$-th unit and $\boldsymbol{u}_{k}^{*}=w_{k} \boldsymbol{y}_{k}^{*}$, where $\boldsymbol{y}_{k}^{*}=\left(y_{k 1}^{*}, \ldots, y_{k p}^{*}\right)^{\top}$ denote the calibrated imputed values having the restriction that $y_{k \ell}^{*}=y_{k \ell}$ if $y_{k \ell}$ is observed and fixed $(k \in A$ and $\ell=1, \ldots, p)$. Following the basic algorithm of Section 2 , consider the problem of finding the $\boldsymbol{u}_{k}^{*}$ satisfying

$$
\sum_{k \in A} \boldsymbol{u}_{k}^{*}=\hat{\boldsymbol{t}}_{0}, \quad \frac{n}{n-1} \sum_{k \in A}\left(\boldsymbol{u}_{k}^{*}-\hat{\boldsymbol{t}}_{0} / n\right)^{\otimes 2}=\hat{\boldsymbol{V}}_{0}
$$

where $\hat{\boldsymbol{t}}_{0}$ denotes a $p$-dimensional vector of target estimates for the population total $\boldsymbol{t}_{\boldsymbol{y}}=\sum_{k \in U} \boldsymbol{y}_{k}, \hat{\boldsymbol{V}}_{0}$ denotes the target estimated variance-covariance matrix of $\hat{\boldsymbol{t}}_{0}$ and $\boldsymbol{a}^{\otimes 2}=\boldsymbol{a} \boldsymbol{a}^{\top}$. To obtain $\hat{\boldsymbol{V}}_{0}$ in the presence of multivariate missing data is a difficult issue. See, e.g., Skinner \& Rao (2002) and Chauvet \& Haziza (2012) for a fully efficient approach in the bivariate case, and Im et al. (2018) and Sang \& Kim (2018) for two fractional imputation methods in the multivariate setting. Below we propose a two-phase calibration procedure, where at the first phase the problem is solved for transformed vectors $\boldsymbol{v}_{k}^{*} \mathrm{~s}(k \in A)$, and at the second phase the results are back-transformed to $\boldsymbol{u}_{k}^{*}$ 's as required. Assume without loss of generality the weighted values

$$
\hat{\boldsymbol{u}}_{k}=w_{k} \hat{\boldsymbol{y}}_{k} \equiv\left(\hat{u}_{k 1}, \ldots, \hat{u}_{k p}\right)^{\top}
$$


satisfy $\sum_{k \in A} \hat{\boldsymbol{u}}_{k}=\hat{\boldsymbol{t}}_{0}$ preserving all the observed and fixed values. This can be achieved for instance by separate imputation of $y_{k \ell}$, for $\ell=1, \ldots, p$, up to Step 1 in Section 2.2.

Let $D$ be the subset of units with all items missing and suppose $m=|D|>0$. Denote by $\hat{\boldsymbol{V}}_{0}=\boldsymbol{P} \boldsymbol{\Lambda} \boldsymbol{P}^{\top}$ the spectral decomposition of $\hat{\boldsymbol{V}}_{0}$, where $\boldsymbol{P}$ is the $p \times p$ orthogonal matrix of the eigenvectors of $\hat{\boldsymbol{V}}_{0}$ and $\boldsymbol{\Lambda}$ is the diagonal matrix of the corresponding eigenvalues. Consider the orthogonal principal components

$$
\hat{\boldsymbol{v}}_{k}=\boldsymbol{P}^{\top}\left(\hat{\boldsymbol{u}}_{k}-\hat{\boldsymbol{t}}_{0} / n\right) \quad(k \in A)
$$

and set

$$
\boldsymbol{v}_{k}^{*}=\hat{\boldsymbol{v}}_{k} \quad(k \in R=A \backslash D) .
$$

Now, choose the $\left\{\boldsymbol{v}_{k}^{*}: k \in D\right\}$ which minimize the squared Frobenius norm

$$
\sum_{k \in D}\left(\boldsymbol{v}_{k}^{*}-\hat{\boldsymbol{v}}_{k}\right)^{\top}\left(\boldsymbol{v}_{k}^{*}-\hat{\boldsymbol{v}}_{k}\right)
$$

subject to

$$
\sum_{k \in D} \boldsymbol{v}_{k}^{*}=-\sum_{k \in R} \hat{\boldsymbol{v}}_{k} \equiv m \overline{\boldsymbol{v}}_{D}^{*}, \quad \sum_{k \in D} \boldsymbol{v}_{k}^{* \otimes 2}=\frac{n-1}{n} \boldsymbol{\Lambda}-\sum_{k \in R} \hat{\boldsymbol{v}}_{k}^{\otimes 2} .
$$

The solution to this constrained optimization problem can be obtained by Theorem 2 below. The proof of this theorem is presented in the Appendix.

Theorem 2. Consider a set of vectors $\hat{\boldsymbol{a}}_{k}(k \in D)$, satisfying $\sum_{k \in D} \hat{\boldsymbol{a}}_{k}=0$ and $\boldsymbol{C}=\sum_{k \in D} \hat{\boldsymbol{a}}_{k}^{\otimes 2}$ being positive definite. Let $\boldsymbol{B}$ be a pre-specified positive definite matrix. Multivariate calibrated vectors that minimize $\sum_{k \in D}\left(\boldsymbol{a}_{k}^{*}-\hat{\boldsymbol{a}}_{k}\right)^{\top}\left(\boldsymbol{a}_{k}^{*}-\hat{\boldsymbol{a}}_{k}\right)$ subjected to

$$
\sum_{k \in D} \boldsymbol{a}_{k}^{*}=0, \quad \sum_{k \in D} \boldsymbol{a}_{k}^{*} \boldsymbol{a}_{k}^{* \top}=\boldsymbol{B}
$$

are $\boldsymbol{a}_{k}^{*}=\boldsymbol{\beta} \hat{\boldsymbol{a}}_{k}$, where $\boldsymbol{\beta}=\boldsymbol{B}^{1 / 2} \boldsymbol{C}^{-1 / 2}$ and $\boldsymbol{B}^{1 / 2}$ and $\boldsymbol{C}^{-1 / 2}$ are the square root and inverse square root matrices of $\boldsymbol{B}$ and $\boldsymbol{C}$, respectively.

In the first phase of the calibration method, we let $\boldsymbol{a}_{k}^{*}=\boldsymbol{v}_{k}^{*}-\overline{\boldsymbol{v}}_{D}^{*}$ and $\hat{\boldsymbol{a}}_{k}=\hat{\boldsymbol{v}}_{k}-\overline{\boldsymbol{v}}_{D}^{*}$ in Theorem 2 If the matrices

$$
\boldsymbol{B} \equiv \frac{n-1}{n} \boldsymbol{\Lambda}-\sum_{k \in R} \hat{\boldsymbol{v}}_{k}^{\otimes 2}-m \overline{\boldsymbol{v}}_{D}^{* \otimes 2}, \quad \boldsymbol{C} \equiv \sum_{k \in D}\left(\hat{\boldsymbol{v}}_{k}-\overline{\boldsymbol{v}}_{D}^{*}\right)^{\otimes 2}
$$

are both positive definite, then the optimal calibrated $\boldsymbol{v}_{k}^{*}$ vectors by Theorem 2 are

$$
\boldsymbol{v}_{k}^{*}=\left(\boldsymbol{I}_{p}-\boldsymbol{\beta}\right) \bar{v}_{D}^{*}+\boldsymbol{\beta} \hat{\boldsymbol{v}}_{k}=\overline{\boldsymbol{v}}_{D}^{*}+\boldsymbol{\beta}\left(\hat{\boldsymbol{v}}_{k}-\overline{\boldsymbol{v}}_{D}^{*}\right) \quad(k \in D),
$$


where $\boldsymbol{I}_{p}$ is the identity matrix of order $p$. Now, from $\sum_{k \in D}\left(\hat{\boldsymbol{v}}_{k}-\overline{\boldsymbol{v}}_{D}^{*}\right)=\sum_{k \in A} \hat{\boldsymbol{v}}_{k}=0$ by [23], it follows that $\sum_{k \in D} \boldsymbol{v}_{k}^{*}=m \overline{\boldsymbol{v}}_{D}^{*}$ and $\sum_{k \in D} \boldsymbol{v}_{k}^{* \otimes 2}=m \overline{\boldsymbol{v}}_{D}^{* \otimes 2}+\boldsymbol{\beta} \boldsymbol{C} \boldsymbol{\beta}^{\top}=m \overline{\boldsymbol{v}}_{D}^{* \otimes 2}+\boldsymbol{B}=(n-1) n^{-1} \boldsymbol{\Lambda}-\sum_{k \in R} \hat{\boldsymbol{v}}_{k}^{\otimes 2}$. We thus enter the second phase of imputation, where we transform the $\boldsymbol{v}_{k}^{*}(k \in D)$ given by (23) and (24) back into

$$
\boldsymbol{u}_{k}^{*}=\boldsymbol{P} \boldsymbol{v}_{k}^{*}+\hat{\boldsymbol{t}}_{0} / n= \begin{cases}\hat{\boldsymbol{u}}_{k}, & (k \in R=A \backslash D), \\ \boldsymbol{P}\left\{\overline{\boldsymbol{v}}_{D}^{*}+\boldsymbol{\beta}\left(\hat{\boldsymbol{v}}_{k}-\overline{\boldsymbol{v}}_{D}^{*}\right)\right\}+\hat{\boldsymbol{t}}_{0} / n, & (k \in D),\end{cases}
$$

which are the final calibrated imputed vectors. The transformation 25 implies that

$$
\sum_{k \in A} \boldsymbol{u}_{k}^{*}=\boldsymbol{P} \sum_{k \in A} \boldsymbol{v}_{k}^{*}+\hat{\boldsymbol{t}}_{0}=\hat{\boldsymbol{t}}_{0}
$$

and

$$
\frac{n}{n-1} \sum_{i \in D}\left(\boldsymbol{u}_{k}^{*}-\hat{\boldsymbol{t}}_{0} / n\right)^{\otimes 2}=\frac{n}{n-1} \boldsymbol{P}\left\{\sum_{k \in R} \hat{\boldsymbol{v}}_{k}^{\otimes 2}+\frac{n-1}{n} \boldsymbol{\Lambda}-\sum_{k \in R} \hat{\boldsymbol{v}}_{k}^{\otimes 2}\right\} \boldsymbol{P}^{\top}=\hat{\boldsymbol{V}}_{0}
$$

as intended.

Notice that the vectors $\boldsymbol{u}_{k}^{*}$ in 25 can be computed directly from $\hat{\boldsymbol{u}}_{k}$ by the linear transformation

$$
\boldsymbol{u}_{k}^{*}=\tilde{\boldsymbol{\alpha}} \mathbb{1}(k \in D)+\left\{\boldsymbol{I}_{p} \mathbb{1}(k \in A \backslash D)+\tilde{\boldsymbol{\beta}} \mathbb{1}(k \in D)\right\} \hat{\boldsymbol{u}}_{k},
$$

where $\tilde{\boldsymbol{\beta}}=\boldsymbol{P} \boldsymbol{\beta} \boldsymbol{P}^{\top}, \tilde{\boldsymbol{\alpha}}=-\left(\boldsymbol{I}_{p}-\tilde{\boldsymbol{\beta}}\right)\left(\sum_{k \in R} \hat{\boldsymbol{u}}_{k}-\hat{\boldsymbol{t}}_{0}\right) / m$ and $\mathbb{1}(\mathcal{A})$ is the indicator function of a set $\mathcal{A}$. All the observed and fixed data values are associated with the units in $R=A \backslash D$, and thus are preserved in the respective $\boldsymbol{u}_{k}^{*}$ values. The $\boldsymbol{u}_{k}^{*}$ and $\hat{\boldsymbol{u}}_{k}$ vectors share the same distance as that of the corresponding $\boldsymbol{v}_{k}^{*}$ and the $\hat{\boldsymbol{v}}_{k}$ vectors, for $k \in D$.

One of the benefits of the proposed calibrated imputation approach above is to facilitate inferences for a nonlinear function of population total vector $\boldsymbol{t}_{y}$. For example, suppose $\sqrt{n}\left(\hat{\boldsymbol{t}}_{0}-\boldsymbol{t}_{y}\right)$ has an asymptotic multivariate normal distribution with mean zero and positive-definite variance-covariance matrix $\boldsymbol{V}$ and that the target variance $\hat{\boldsymbol{V}}_{0}$ is consistent for the variance of $\hat{\boldsymbol{t}}_{\boldsymbol{y}}$. Thus, an approximate $100(1-\alpha) \%$ confidence interval for $g\left(t_{y}\right)$, where $g: \mathbb{R}^{p} \rightarrow \mathbb{R}$ is smooth and has nonzero gradient $\nabla g(\cdot)$ at $\boldsymbol{t}_{y}$, is

$$
g\left(\hat{\boldsymbol{t}}_{y I}\right) \pm z_{1-\alpha / 2}\left[\nabla^{\top} g\left(\hat{\boldsymbol{t}}_{y I}\right)\left\{\frac{n}{n-1} \sum_{k \in A}\left(\boldsymbol{u}_{k}^{*}-\hat{\boldsymbol{t}}_{0} / n\right)^{\otimes 2}\right\} \nabla g\left(\hat{\boldsymbol{t}}_{y I}\right)\right]^{1 / 2},
$$

where $\hat{\boldsymbol{t}}_{y I}=\sum_{k \in A} \boldsymbol{u}_{k}^{*}=\hat{\boldsymbol{t}}_{0}$, which is Wald-type interval that could alternatively be computed by

$$
g\left(\hat{\boldsymbol{t}}_{y I}\right) \pm z_{1-\alpha / 2}\left[\hat{v}_{F}\left\{g\left(\hat{\boldsymbol{t}}_{y I}\right)\right\}\right]^{1 / 2}
$$


where

$$
\hat{v}_{F}\left\{g\left(\hat{\boldsymbol{t}}_{y I}\right)\right\}=\frac{n}{n-1} \sum_{k \in A}\left(\boldsymbol{u}_{g k}^{*}-\hat{\boldsymbol{t}}_{g 0} / n\right)^{\otimes 2},
$$

$\boldsymbol{u}_{g k}^{*}=\nabla^{\top} g\left(\hat{\boldsymbol{t}}_{y I}\right) \boldsymbol{u}_{k}^{*}$ and $\hat{\boldsymbol{t}}_{g 0}=\nabla^{\top} g\left(\hat{\boldsymbol{t}}_{y I}\right) \hat{\boldsymbol{t}}_{0}$. The use of the estimate $\hat{v}_{F}\left\{g\left(\hat{\boldsymbol{t}}_{y I}\right)\right\}$ above does not merely simplifies the computation of the estimated variance of $g\left(\hat{\boldsymbol{t}}_{y I}\right)$ by a complete sample formula, but it also prevents the secondary users to having to apply a specific variance estimation method and corresponding software to estimate the variance of $g\left(\hat{\boldsymbol{t}}_{y I}\right)$.

\section{5 | CONCLUDING REMARKS}

In this paper we propose a calibrated imputation approach to be used routinely in the preparation of imputed sample survey data, under a stratified multistage sampling design. It allows secondary users to estimate specified population parameters and associated variances and covariances by simple complete-sample formulae, regardless of how complicatedly it may be when these are to be derived from the original incomplete data. Domain estimation can be accommodated in addition. The approach avoids the need to disseminate multiple imputed (or replicated) datasets.

A topic for future research is the imputation of categorical variables. The fractional imputation of Kim \& Fuller (2004) provides a fully efficient imputation method, where $K$ imputed values are created for a $K$-category variable. Favre et al. (2005) propose a fully efficient single-sample imputation method, under which a categorical variable is treated as a vector of dummyindicators during the imputation. However, variance estimation under these methods may require a different approach than by using simple complete-sample formulae. It seems possible to adapt the multivariate calibrated imputation approach in this paper, where the imputed values of the dummy-vector can be continuous instead of just 0 and 1 , given the target estimates of the population totals of each category and the associated variances and covariances.

Another research topic of interest is calibrated imputation for quantile estimation. For instance, given the target quantile estimate $\hat{t}_{\alpha}$ and its variance estimate $\hat{v}_{\alpha}$, for $0<\alpha<1$. It may be possible to adapt the proposed algorithm in Section 2.2 . as follows. At Step 1, adjust the initial imputed values $\left\{\tilde{y}_{k}: k \in A_{m}\right\}$ to $\left\{\hat{y}_{k}: k \in A_{m}\right\}$, which satisfy

$$
\sum_{k \in A_{r}} w_{i} I\left(y_{k} \leqslant \hat{t}_{\alpha}\right)+\sum_{k \in A_{m}} w_{i} I\left(\hat{y}_{k} \leqslant \hat{t}_{\alpha}\right)=\alpha \sum_{k \in A} w_{i}
$$

Then, at Step 2, adjust the negative and positive deviations $\hat{y}_{k}-\hat{t}_{\alpha}$, for $k \in A_{m}$, while maintaining their signs to satisfy a suitably chosen complete-sample variance formula. 


\section{ACKNOWLEDGMENTS}

This research was developed at the Southampton Statistical Sciences Research Institute of the University of Souhampton, UK. The research of the first author was supported by a grant from the Brazilian Research Council (CNPq). We are thankful to the Associate Editor and two anonymous referees for their useful comments.

\section{References}

Beaumont, J.-F. (2005). Calibrated imputation in surveys under a quasi-model-assisted approach. J. R. Stat. Soc. Ser. B Stat. Methodol., 67(3), 445-458.

Chambers, R. L., \& Ren, R. (2004). Outlier robust imputation of survey data. In JSM Proceedings, the Survey Research Methods Section, 3336-3344, Alexandria, VA: American Statistical Association.

Chauvet, G., Deville, J.-C., \& Haziza, D. (2011). On balanced random imputation in surveys. Biometrika, 98(2), 459-471.

Chauvet, G., \& Haziza, D. (2012). Fully efficient estimation of coefficients of correlation in the presence of imputed survey data. Canad. J. Statist., $\underline{40}(1), 124-149$.

Chen, J., Rao, J. N. K., \& Sitter, R. R. (2000). Efficient random imputation for missing data in complex surveys. Statist. Sinica, 10(4), 1153-1169.

Deville, J.-C., \& Särndal, C.-E. (1992a). Calibration estimators in survey sampling. J. Amer. Statist. Assoc., 87, 376-382.

Deville, J.-C., \& Särndal, C.-E. (1994b). Variance estimation for the regression imputed horvitz-thompson estimator. Journal of Official Statistics, 10, 381-394.

Favre, A.-C., Matei, A., \& Tillé, Y. (2005). Calibrated random imputation for qualitative data. J. Statist. Plann. Inference, $\underline{128}(2), 411-425$.

Fay, R. E. (1991). A design-based perspective on missing data variance. In Proceedings of the 1991 Annual Research Conference, 429-440, Washington, D.C.: U.S. Dept. of Commerce, Bureau of the Census. 
Fay, R. E. (1992). When are inferences from multiple imputation valid? In JSM Proceedings, the Survey Research Methods Section, 227-232, Alexandria, VA: American Statistical Association.

Fuller, W. A., \& Kim, J. K. (2005). Hot deck imputation for the response model. Survey Methodology, 31(2), 139-149.

Gelein, B., Haziza, D., \& Causeur, D. (2014). Preserving relationships between variables with MIVQUE based imputation for missing survey data. J. Multivariate Anal., 131(0), $197-208$.

Ghosh, M. (1992). Constrained bayes estimation with applications. J. Amer. Statist. Assoc., 87(418), 533-540.

Haziza, D. (2009). Imputation and inference in the presence of missing data. In D. Pfeffermann and C. R. Rao (Eds.), Handbook of Statistics, Volume 29A, Sample surveys: Design, methods and applications (pp. 215-246). Amsterdam: Elsevier.

Horvitz, D., \& Thompson, D. (1952). A generalization of sampling without replacement from a finite universe. J. Amer. Statist. Assoc., 47, 663-685.

Im, J., Cho, I. H., \& Kim, J. K. (2018). FHDI: An R package for fractional hot deck imputation. The R Journal, 10(1), 140-154.

Kalton, G., \& Kasprzyk, D. (1982). Imputing for missing survey responses. In JSM Proceedings, the Survey Research Methods Section, 22-31, Alexandria, VA: American Statistical Association.

Kim, J. K. (2001a). Variance estimation afeter imputation. Survey Methodology, 27(1), 75-83.

Kim, J. K. (2011b). Parametric fractional imputation for missing data analysis. Biometrika, 98(1), 119-132.

Kim, J. K., \& Fuller, W. (2004). Fractional hot deck imputation. Biometrika, 91(3), 559-578.

Kim, J. K., \& Rao, J. N. K. (2009). A unified approach to linearization variance estimation from survey data after imputation for item nonresponse. Biometrika, 96(4), 917-932.

Lanke, J. (1983). Hot deck imputation techniques that permit standard methods for assessing precision of estimates. Statistical

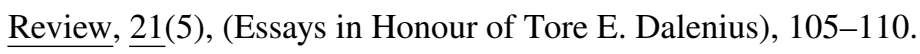

Meng, X.-L. (1994). Multiple-imputation inferences with uncongenial sources of input. Statist. Sci., 9(4), 538-558.

Rao, J. N. K. (1996). On variance estimation with imputed survey data. J. Amer. Statist. Assoc., 91(434), 499-506. 
Rao, J. N. K., \& Shao, J. (1992). Jackknife variance estimation with survey data under hot deck imputation. Biometrika, 79, $811-822$.

Rubin, D. B. (1978a). Multiple imputations in sample surveys: A phenomenological Bayesian approach to nonresponse. In JSM Proceedings, the Survey Research Methods Section, 20-28, Alexandria, VA: American Statistical Association.

Rubin, D. B. (1987b). Multiple imputation for nonresponse in surveys. Hoboken, NJ: Wiley.

Rubin, D. B. (1996c). Multiple imputation after 18+ years. J. Amer. Statist. Assoc., 91(434), 473-489.

Rubin, D. B., \& Schenker, N. (1986). Multiple imputation for interval estimation from simple random samples with ignorable nonresponse. J. Amer. Statist. Assoc., 81(394), 366-374.

Sang, H., \& Kim, J. K. (2018). Semiparametric fractional imputation using gaussian mixture models for handling multivariate missing data. Unpublished manuscript. arXiv:1809.05976. Retrieved from https://arxiv.org/abs/1809.05976.

URL https://arxiv.org/abs/1809.05976

Särndal, C.-E. (1992). Methods for estimating the precision of survey estimates when imputation has been used. Survey $\underline{\text { Methodology, } 18,241-252 .}$

Sedransk, J. (1985). The objectives and practice of imputation. In Proceedings of the First Annual Research Conference, 445-452, Washington, D.C.: Bureau of the Census.

Shao, J., \& Sitter, R. R. (1996). Bootstrap for Imputed Survey Data. J. Amer. Statist. Assoc., 91(435), 1278-1288.

Shao, J., \& Steel, P. (1999). Variance estimation for survey data with composite imputation and nonnegligible sampling fractions. J. Amer. Statist. Assoc., 94(445), 254-265.

Skinner, C., \& Rao, J. (2002). Jackknife variance estimation for multivariate statistics under hot-deck imputation from common donors. J. Statist. Plann. Inference, $102(1), 149-167$.

Skinner, C. J. (1989). Introduction to part A. In C. J. Skinner, D. Holt, and T. M. F. Smith (Eds.), Analysis of complex surveys (pp. 23-58). Chichester: Wiley.

Wolter, K. M. (2007). Introduction to variance estimation (2nd ed.). New York: Springer. 


\section{SUPPORTING INFORMATION}

The supporting information, available as part of the online article, provides numerical illustrations for the proposed approach to estimate a population total and to preserve variability. Examples consider element sampling, stratified multistage sampling and also calibration of imputed multivariate data.

\section{APPENDIX}

\section{A PROOF OF THEOREM 1}

The proof of Theorem 1 is shown below. In the special case of $d_{k}=1$, the problem addressed by Theorem 1 reduces essentially to that of the small-area constrained Bayes estimators (Ghosh, 1992, Theorem 1), which minimize an unweighted quadratic function of the prediction errors. The two constraints in Ghosh (1992. Theorem 1), despite being formulated in a small area estimation setting, are equivalent in form to (8).

Proof. Notice that

$$
\sum_{k \in D} d_{k}\left(a_{k}-\hat{a}_{k}\right)^{2}=t_{2}-2 \sum_{k \in D} d_{k} a_{k} \hat{a}_{k}+\hat{t}_{2}
$$

Since $t_{2}$ and $\hat{t}_{2}$ are positive and fixed, one needs to maximize $\sum_{k \in D} d_{k} a_{k} \hat{a}_{k}$ or, equivalently, $\sum_{k \in D} q_{k} a_{k} \hat{a}_{k}$, where $q_{k}=d_{k} / t_{0}$ and $\sum_{k \in D} q_{k}=1$. Consider then $\left(q_{1}, \ldots, q_{m}\right)$ as a discrete distribution function, denoted by $\mathcal{F}_{q}$, which puts probability $q_{k}$ on each pair of $\left(a_{k}, \hat{a}_{k}\right)$, for $k \in D$. We have $\sum_{k \in D} q_{k} a_{k} \hat{a}_{k}=E_{q}\left(a_{k} \hat{a}_{k}\right)$, i.e., the expectation over $\mathcal{F}_{q}$. Notice that $E_{q}\left(a_{k}\right)=\sum_{k \in D} q_{k} a_{k}=t_{1} / t_{0}$ and $E_{q}\left(\hat{a}_{k}\right)=t_{1} / t_{0}$ are both fixed, as well as $V_{q}\left(a_{k}\right)=t_{2} / t_{0}-\left(t_{1} / t_{0}\right)^{2}$ and $V_{q}\left(\hat{a}_{k}\right)=\hat{t}_{2} / t_{0}-\left(t_{1} / t_{0}\right)^{2}$. Thus, the maximum of $E_{q}\left(a_{k} \hat{a}_{k}\right)$ is the same as that of $\operatorname{Cov}_{q}\left(a_{k}, \hat{a}_{k}\right)$, which is given when the correlation between $a_{k}$ and $\hat{a}_{k}$ is equal to 1 over $\mathcal{F}_{q}$, i.e., $a_{k}=\alpha+\beta \hat{a}_{k}$ with probability one, for some constants $\alpha$ and $\beta$. Solving $(\alpha, \beta)$ for $(8)$ yields then $\alpha=(1-\beta) t_{1} / t_{0}$ and $\beta=\left[\left(t_{2}-t_{1}^{2} / t_{0}\right) /\left(\hat{t}_{2}-t_{1}^{2} / t_{0}\right)\right]^{1 / 2}$. This completes the proof. 


\section{B PROOF OF THEOREM 2}

Proof. Let the Lagrangian of this constrained optimisation problem be

$$
L=\frac{1}{2} \sum_{k \in D}\left(\tilde{\boldsymbol{a}}_{k}^{*}-\boldsymbol{a}_{k}^{*}\right)^{\top}\left(\tilde{\boldsymbol{a}}_{k}^{*}-\boldsymbol{a}_{k}^{*}\right)-\boldsymbol{\psi}^{T} \sum_{k \in D} \tilde{\boldsymbol{a}}_{k}^{*}-\mathbf{1}^{\top}\left[\boldsymbol{\Lambda} \circ\left(\sum_{k \in D} \tilde{\boldsymbol{a}}_{k}^{*} \tilde{\boldsymbol{a}}_{k}^{* \top}-\boldsymbol{S}_{D}^{*}\right)\right] \mathbf{1}
$$

for some vector $\boldsymbol{\psi}$ and matrix $\Lambda$, where $\mathbf{1}$ denotes a vector of ones and "o" denotes the Hadamard (element-wise) product of two matrices. We have

$$
\left(\partial L / \partial \tilde{\boldsymbol{a}}_{k}^{*}\right)^{\top}=\left(\tilde{\boldsymbol{a}}_{k}^{*}-\boldsymbol{a}_{k}^{*}\right)-\boldsymbol{\psi}-\left(\boldsymbol{\Lambda}+\boldsymbol{\Lambda}^{\top}\right) \tilde{\boldsymbol{a}}_{k}^{*}=0 \quad \Rightarrow \quad \tilde{\boldsymbol{a}}_{k}^{*}=\left[\boldsymbol{I}_{p}-\left(\boldsymbol{\Lambda}+\boldsymbol{\Lambda}^{\top}\right)\right]^{-1}\left(\boldsymbol{a}_{k}^{*}+\boldsymbol{\psi}\right)
$$

provided $\boldsymbol{W}=\left[\boldsymbol{I}_{p}-\left(\boldsymbol{\Lambda}+\boldsymbol{\Lambda}^{\top}\right)\right]^{-1}$ exists. Notice that $\boldsymbol{W}$ is symmetric. Substitution of $\tilde{\boldsymbol{a}}_{k}^{*}=\boldsymbol{W}\left(\boldsymbol{a}_{k}^{*}+\boldsymbol{\psi}\right)$ into $\sum_{k \in D} \boldsymbol{a}_{k}^{*}=0$ yields $\boldsymbol{\psi}=0$. Next, substitution of $\tilde{\boldsymbol{a}}_{k}^{*}=\boldsymbol{W} \boldsymbol{a}_{k}^{*}$ into $\sum_{k \in D} \boldsymbol{a}_{k}^{*} \boldsymbol{a}_{k}^{* \top}=\boldsymbol{B}$ yields

$$
\boldsymbol{B} \boldsymbol{B}^{\top}=\sum_{D} \boldsymbol{W} \boldsymbol{a}_{k}^{*} \boldsymbol{a}_{k}^{* \top} \boldsymbol{W}=\boldsymbol{W} \boldsymbol{C} \boldsymbol{C}^{\top} \boldsymbol{W} \quad \Rightarrow \quad \boldsymbol{W}=\boldsymbol{B} \boldsymbol{C}^{-1}
$$

This completes the proof. 